\title{
Light Scattering and Phase Separation Studies on Cyclohexane Solutions of Six-Arm Star Polystyrene ${ }^{\dagger}$
}

\author{
By Yoshiko TASAKA, Mitsuhiro OKUMOTO, Yo NAKAMURA, ${ }^{* \dagger \dagger}$ and Takashi NORISUYE
}

Light scattering and phase separation experiments were performed for four six-arm star polystyrene (6SPS) samples with weight-average molecular weights $M_{\mathrm{w}}$ of $9.62 \times 10^{4}$ to $1.16 \times 10^{6}$ in cyclohexane below the theta temperature $\left(34.5^{\circ} \mathrm{C}\right)$. From the former experiment, the apparent second virial coefficient $J$ was obtained as functions of the polymer volume fraction $\phi$ and temperature $T$, along with the spinodals. In the latter experiment, the concentrations of coexisting two phases were determined as functions of $T$. The critical point $T_{\mathrm{c}}$ determined from the coexisting curve was lower than that for fourarm star polystyrene (4SPS) when compared at the same $M_{\mathrm{w}}$. As was the case for 4SPS and linear polystyrene in cyclohexane, $J$ for 6SPS at each $T$ in a region of large $\phi$ was represented by a universal function of $\phi / P^{0.1}$ regardless of $P$ (the volume of the polymer chain relative to that of the solvent molecule), although it differed from the common function previously found for the other two types of polystyrene. It was concluded that the solubility of 6SPS higher than that of 4SPS in cyclohexane as indicated by the lower critical point is attributable to the chain-end effect.

KEY WORDS: Star Polymer / Phase Separation / Chemical Potential / Binodal / Spinodal / Critical Point / Polystyrene /

It is known that the phase separation temperature for star polymers in poor solvents are lower than that for the corresponding linear polymer with the same molecular weight and the same chemical structure. ${ }^{1-6}$ This cannot be explained by theories invoking the Flory-Huggins type ${ }^{7}$ mean-field approximation, but at present, there exists no molecular theory that explains the phase behavior of branched molecules. Recent Monte Carlo simulation results also fail to describe the experimental data. ${ }^{8,9}$ In this situation, a phenomenological approach to the problem may be useful as an alternate. $^{10-12}$

In our previous work, Terao et al. $^{4}$ carried out light scattering and phase separation experiments on four-arm star polystyrene (PS) in cyclohexane below the theta point $\Theta$ $\left(34.5^{\circ} \mathrm{C}\right)$. Their light scattering data showed that the apparent second virial coefficient $J$ at a fixed temperature $T$ in a region of high polymer volume fraction $\phi$ can be represented by a universal function of $\phi / P^{0.1}$ regardless of molecular weight and that the same function is also applicable to linear PS in cyclohexane, where $P$ is the relative chain length (the volume of the polymer chain relative to that of the solvent molecule). The difference in $J$ between four-arm star and linear PS's appeared only at low $\phi$. Thus, it was concluded that the difference in the chemical potential of the solvent in the low $\phi$ region is responsible for the difference in phase separation behavior between the two polymers.

The present study was undertaken as an extension of the previous work to six-arm star PS in cyclohexane below $\Theta$ to examine the $J$ function behavior and the phase diagrams in relation to four-arm star and linear PS's. Light scattering and phase separation data obtained as functions of $T$ and $P$ and their analyses are presented below, along with comparison of the experimental coexisting curves, critical points, and spinodals with those computed from the $J$ functions.

\section{EXPERIMENTAL}

\section{Polymer Samples and Preparation of Solutions}

Six-arm star PS samples, 6S17, 6S36, 6S85, and 6S190 with weight-average molecular weights $M_{\mathrm{w}}$ of $9.62 \times 10^{4}, 21.0 \times$ $10^{4}, 50.1 \times 10^{4}$, and $116 \times 10^{4}$, respectively, were chosen from those used previously for dilute solution studies. ${ }^{13,14}$ These samples, synthesized by a coupling reaction of anionically polymerized living polystyrene, were well fractionated and their weight to number-average molecular weight ratios were less than 1.04. The ratio of $M_{\mathrm{w}}$ of each sample to that for its arm was $6 \pm 0.3$, ensuring that each molecule has almost exactly six arms with very narrow length distribution. ${ }^{13,14}$

The chosen samples were dried for more than $24 \mathrm{~h}$ in vacuo and dissolved into cyclohexane, which had been dried over sodium metal, refluxed for $4 \mathrm{~h}$, and fractionally distilled. Solutions for light scattering measurements were optically clarified with a Teflon filter of $0.5 \mu \mathrm{m}$ pore size. The volume fraction $\phi$ of the solute was calculated from the gravimetrically determined weight fraction $w$ by

$$
\phi=v_{\mathrm{p}} w /\left[v_{\mathrm{p}} w+v_{0}(1-w)\right]
$$

with the specific volume of cyclohexane $v_{0}=1.292 \mathrm{~cm}^{3} \mathrm{~g}^{-1}$ and that of PS $v_{\mathrm{p}}=0.934 \mathrm{~cm}^{3} \mathrm{~g}^{-1}$ (both at $25^{\circ} \mathrm{C}$ ); where $v_{0}$ and $v_{\mathrm{p}}$ were regarded as temperature independent.

\footnotetext{
Department of Macromolecular Science, Osaka University, 1-1 Machikaneyama-cho, Toyonaka 560-0043, Japan

*To whom correspondence should be addressed (E-mail: yonaka@molsci.polym.kyoto-u.ac.jp).

Dedicated to the late Professor Hiroshi Fujita.

Present address: Department of Polymer Chemistry, Kyoto University, Katsura, Kyoto 615-8510, Japan.
} 


\section{Light Scattering Measurements}

Intensity measurements were made on a Fica-50 light scattering photometer with vertically polarized incident light of $\lambda_{0}$ (the wavelength) $=546 \mathrm{~nm}$ in an angular range from 30 to $150^{\circ}$. The solution temperature was kept within $\pm 0.05^{\circ} \mathrm{C}$. Neutral filters with known transmittance were used when the scattering intensity exceeded the limit of the detector. Since, considerable effects of absorption and multiple scattering on scattering intensity were observed in our previous study, ${ }^{4}$ the measured (or apparent) reduced intensity $R_{\theta \text {,app }}$ at scattering angle $\theta$ was corrected for them according to the following procedure of Terao et al. ${ }^{4}$ by

The true reduced scattering intensity $R_{\theta}$ is related to $R_{\theta \text {,app }}$

$$
R_{\theta}=R_{\theta, \text { app }} \exp (2.303 \gamma l c+\tau l)-f(\theta) \tau^{2}
$$

where $\gamma$ is the absorption coefficient $\left(0.08 \mathrm{~cm}^{2} \mathrm{~g}^{-1}\right.$ at $\left.546 \mathrm{~nm}\right)$, $l$ is the path length of light in the cell $(2.2 \mathrm{~cm}), c$ is the polymer mass concentration, $\tau$ is the turbidity related to $R_{90}$ by

$$
R_{90}=3 \tau / 8 \pi
$$

and $f(\theta)$ is given by

$$
f(\theta)=0.07+0.09 \sin \theta
$$

Substitution of experimental values for $R_{90 \text {,app }}$ and $f(90)$ into eq 2 , followed by numerical solution with eq 3 , gives $\tau$, which, in turn, allows us to evaluate $R_{\theta}$ from eq 2 with experimental $R_{\theta \text {,app }}$. We note that near the critical point, the difference between $R_{\theta}$ and $R_{\theta \text {,app }}$ arises predominantly from multiple scattering and amounts to $90 \%$ for sample 6S36 at $\phi=0.00796$ and $T=22{ }^{\circ} \mathrm{C}$ (see Table II for the critical point).

The reduced scattering intensity at $\theta=0$ denoted by $R_{0}$ is related to the chemical potential of the solvent $\mu_{0}$ by the following equation:

$$
\frac{K^{\prime} \phi}{R_{0}}=-\frac{1}{R T}\left(\frac{\partial \mu_{0}}{\partial \phi}\right)
$$

Here, $R$ is the gas constant and $K^{\prime}$ is a constant defined by

$$
K^{\prime}=\frac{4 \pi^{2} n^{2} V_{0}}{N_{\mathrm{A}} \lambda_{0}{ }^{4}}\left(\frac{\partial n}{\partial \phi}\right)^{2}
$$

with $V_{0}$ the molar volume of the solvent ( $v_{0}$ multiplied by its molecular weight), $N_{\mathrm{A}}$ the Avogadro constant, and $n$ the refractive index of the solution. The specific refractive index increment $\partial n / \partial \phi$ was calculated from the previously determined $\partial n / \partial c$ for six-arm star PS in cyclohexane ${ }^{13}$ as a function of temperature and molecular weight; its concentration dependence was ignored.

\section{Phase Separation Experiments}

Phase separation experiments were carried out in a Brice type cell composed of two triangular sections; one is filled with a test solution and the other with the pure solvent. It took $5 \mathrm{~h}$ to $2 \mathrm{~d}$ for equilibration at a temperature kept within $\pm 0.01^{\circ} \mathrm{C}$. The solute concentration in each phase-separated solution was determined by differential refractometery as described previ-

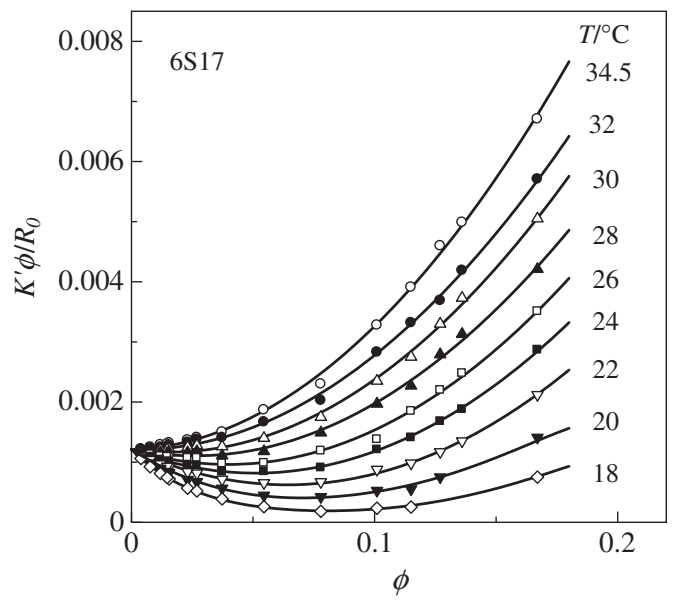

Figure 1. Scattering intensity data for six-arm star polystyrene sample 6S17 at different temperatures indicated.

ously. ${ }^{4}$ The instrument was calibrated with cyclohexane solutions of sample $6 \mathrm{~S} 17$ with different $w$ 's lower than 0.15 at temperatures between 25 and $35^{\circ} \mathrm{C}$.

\section{RESULTS}

\section{Apparent Second Virial Coefficient}

Figure 1 shows $K^{\prime} \phi / R_{0}$ plotted against $\phi$ for six-arm star PS sample $6 \mathrm{~S} 17$ at the indicated temperatures. All the curves fitting the data points at the respective $T$ bend upward and have a common intercept $1 / P$ at $\phi=0$, where $P$ is given by

$$
P=v_{\mathrm{p}} M_{\mathrm{w}} / V_{0}
$$

The concentration dependence of the apparent second virial coefficient $J$ defined by

$$
J=\left[\left(K^{\prime} \phi / R_{0}\right)-(1 / P)\right] / 2 \phi
$$

is illustrated for four samples in Figure 2. The data points at each $T$ (i.e., for a given symbol) follow a weakly sigmoidal curve as was observed for linear and four-arm star PS' s. ${ }^{4,11}$ The intercept (denoted below as $J_{0}$ ) and the initial slope of each curve are related to the second virial coefficient $A_{2}$ and the third virial coefficient, respectively, whose numerical data and some discussions are given in ref 13 . The relation between $J_{0}$ and $A_{2}$ is represented by

$$
J_{0}=\left(V_{0} / v_{\mathrm{p}}^{2}\right) A_{2}
$$

In the previous studies, $J_{0}$ 's for linear ${ }^{11}$ and four-arm star ${ }^{4}$ PS's in cyclohexane below $\Theta$ were expressed approximately by functions of $(1-\Theta / T)$ regardless of $M_{\mathrm{w}}$ in the range examined. For six-arm star PS, however, this is not the case, because $A_{2 \Theta}$ (i.e., $A_{2}$ at $\Theta$ ) in cyclohexane becomes positive and increases with decreasing $M_{\mathrm{w}} \cdot{ }^{13}$ It can be expressed as

$$
A_{2 \Theta}=2.5 / M_{\mathrm{w}}
$$

in units of $\mathrm{cm}^{3} \mathrm{~mol} \mathrm{~g}^{-2}$.

The positive $A_{2}$ at $\Theta$ may be considered due to the effect from the chain ends. ${ }^{15-17}$ We remove this effect on $J$ by subtracting $J_{0 \Theta}$ (the value of $J_{0}$ at $\Theta$ ) and thus have 

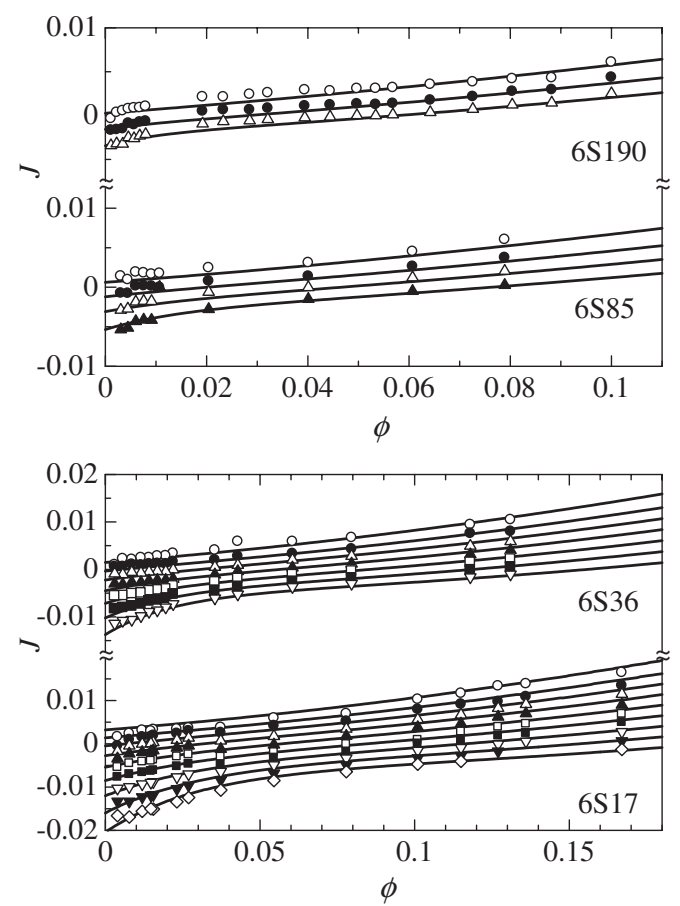

Figure 2. Apparent second virial coefficient $J$ plotted against polymer volume fraction $\phi$ for six-arm star polystyrene samples at temperatures of $34.5,32,30,28,26,24,22,20$, and $18^{\circ} \mathrm{C}$ from top to bottom.

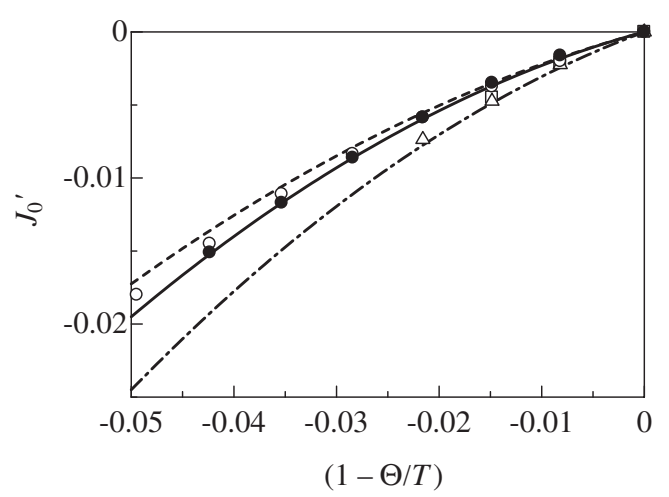

Figure 3. Temperature dependence of reduced second virial coefficient: unfilled circles, 6S17; filled circles, 6S36; triangles, 6S85; squares, 6S190. Solid, dashed, and dot-dashed lines represent the calculated values ${ }^{4}$ for six-arm star, four-arm star, and linear polystyrenes, respectively.

$$
J_{0}{ }^{\prime}=J_{0}-J_{0 \Theta}
$$

Note that $J_{0 \Theta}$ is obtained from eq 9 with eq 10 .

In Figure 3, the $J_{0}{ }^{\prime}$ values calculated from $A_{2}$ data in ref 13 are plotted against $(1-\Theta / T)$. The data points are approximately fitted by a solid curve, which is expressed as

$$
J_{0}{ }^{\prime}=0.19(1-\Theta / T)-4.0(1-\Theta / T)^{2}
$$

The dashed and dot-dashed lines represent the relations for four-arm star ${ }^{4}$ and linear ${ }^{11}$ PS's $\left(J_{0}^{\prime}=J_{0}\right.$ for these polymers), respectively. The lines for the star polymers are

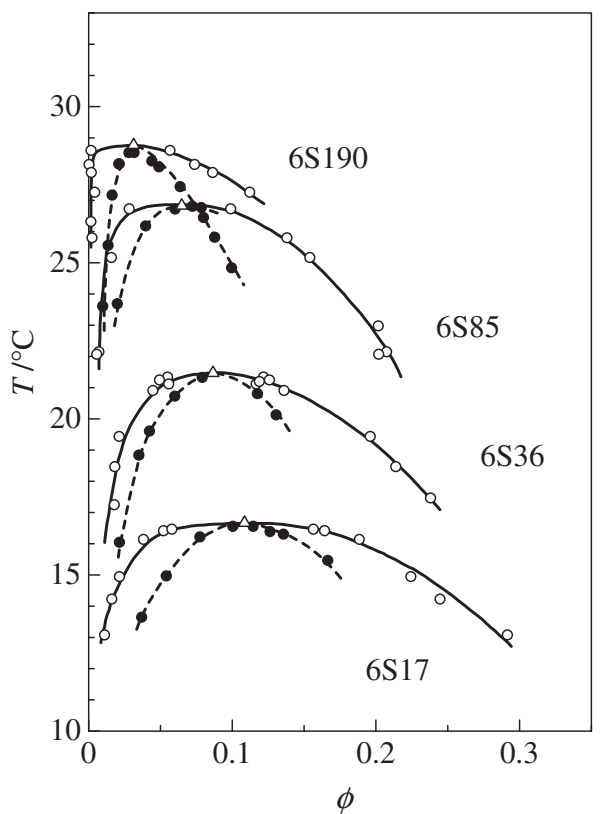

Figure 4. Phase diagrams for six-arm star polystyrene samples in cyclohexane. Unfilled circles, filled circles, and triangles represent the coexisting phase concentrations, spinodals, and critical points, respectively. Lines are eye guides.

Table I. Polymer volume fractions of coexisting phases

\begin{tabular}{|c|c|c|c|c|c|}
\hline \multicolumn{3}{|c|}{$6 \mathrm{~S} 17$} & \multicolumn{3}{|c|}{$6 S 36$} \\
\hline \multicolumn{3}{|c|}{$M_{w}=96,200$} & \multicolumn{3}{|c|}{$M_{\mathrm{w}}=210,000$} \\
\hline$T /{ }^{\circ} \mathrm{C}$ & $\phi^{\prime}$ & $\phi^{\prime \prime}$ & $T /{ }^{\circ} \mathrm{C}$ & $\phi^{\prime}$ & $\phi^{\prime \prime}$ \\
\hline 16.45 & 0.0584 & 0.1569 & 21.32 & 0.0554 & 0.1222 \\
\hline 16.40 & 0.0526 & 0.1647 & 21.23 & 0.0498 & 0.1263 \\
\hline 16.12 & 0.0386 & 0.1889 & 21.18 & - & 0.1193 \\
\hline 14.93 & 0.0219 & 0.2248 & 21.10 & 0.0562 & 0.1171 \\
\hline 14.21 & 0.0166 & 0.2451 & 20.89 & 0.0452 & 0.1365 \\
\hline \multirow[t]{4}{*}{13.06} & 0.0115 & 0.2920 & 19.42 & 0.0217 & 0.1965 \\
\hline & & & 18.45 & 0.0187 & 0.2143 \\
\hline & & & 17.45 & - & 0.2385 \\
\hline & & & 17.23 & 0.0183 & - \\
\hline \multicolumn{3}{|c|}{$6 S 85$} & \multicolumn{3}{|c|}{$6 S 190$} \\
\hline \multicolumn{3}{|c|}{$M_{\mathrm{w}}=501,000$} & \multicolumn{3}{|c|}{$M_{\mathrm{w}}=1,160,000$} \\
\hline $\mathrm{T} /{ }^{\circ} \mathrm{C}$ & $\phi^{\prime}$ & $\phi^{\prime \prime}$ & $T /{ }^{\circ} \mathrm{C}$ & $\phi^{\prime}$ & $\phi^{\prime \prime}$ \\
\hline 26.71 & 0.0287 & 0.0994 & 28.58 & 0.0021 & 0.0571 \\
\hline 25.15 & 0.0163 & 0.1545 & 28.13 & 0.0007 & 0.0742 \\
\hline 22.96 & - & 0.2021 & 27.87 & 0.0023 & 0.0868 \\
\hline 22.13 & 0.0076 & 0.2079 & 27.24 & 0.0047 & 0.1126 \\
\hline 18.59 & 0.0097 & 0.2631 & 26.30 & 0.0020 & - \\
\hline 16.95 & 0.0129 & 0.2741 & 25.78 & 0.0028 & - \\
\hline
\end{tabular}
for six-arm star polystyrene in cyclohexane

very close to each other and far above that for the linear polymer.

\section{Phase Diagram}

The concentrations of coexisting phases are shown by unfilled circles in Figure 4 and summarized in Table I, in which $\phi^{\prime}$ and $\phi^{\prime \prime}$ denote the polymer volume fractions in the dilute and concentrated phases, respectively. 
Table II. Critical temperature and concentration for six-arm star polystyrene in cyclohexane

\begin{tabular}{ccc}
\hline Sample & $T_{\mathrm{c}} /{ }^{\circ} \mathrm{C}$ & $\boldsymbol{\phi}_{\mathrm{c}}$ \\
\hline $6 \mathrm{~S} 17$ & $16.7 \pm 0.1$ & $0.108 \pm 0.001$ \\
$6 \mathrm{~S} 36$ & $21.4 \pm 0.1$ & $0.086 \pm 0.002$ \\
$6 \mathrm{~S} 85$ & $26.8 \pm 0.1$ & $0.066 \pm 0.002$ \\
$6 \mathrm{~S} 190$ & $28.7 \pm 0.1$ & $0.031 \pm 0.002$
\end{tabular}

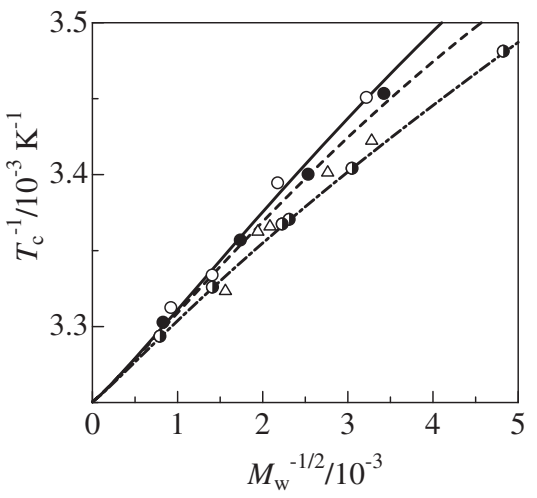

Figure 5. Reciprocal of the critical temperature plotted against $M_{w}^{-1 / 2}$. Unfilled circles, filled circles, triangles, and half-filled circles represent the data for six-arm star, four-arm star, ${ }^{4}$ three-arm star, ${ }^{1}$ and linear ${ }^{19-22}$ polystyrenes, respectively, in cyclohexane.

The critical temperature $T_{\mathrm{c}}$ was determined by extrapolation of the plot of $\Delta \phi\left[=\left(\phi^{\prime}-\phi^{\prime \prime}\right)^{1 / \beta}\right]$ against $T$ to $\Delta \phi=0,{ }^{3}$ with the critical exponent $\beta$ taken to be the value 0.327 from the three-dimensional Ising model. ${ }^{18}$ The critical concentration $\phi_{\mathrm{c}}$ was estimated by extrapolation of the $\left(\phi^{\prime}+\phi^{\prime \prime}\right) / 2$ vs. $\left(T_{\mathrm{c}}-T\right)$ plot to $\left(T_{\mathrm{c}}-T\right)=0$. The critical points thus obtained are indicated by the triangles in Figure 4 and summarized in Table II.

The molecular weight dependence of $T_{\mathrm{c}}^{-1}$ is illustrated in Figure 5, in which the unfilled, filled, and half-filled circles represent the data for six-arm star (present data) four-arm star, ${ }^{4}$ and linear ${ }^{19-22}$ PS's, respectively, and the triangles, those for three-arm star PS, ${ }^{1}$ all in cyclohexane. Comparison of these data at the same $M_{\mathrm{w}}$ reveals that $T_{\mathrm{c}}^{-1}$ increases with increasing arm number. In other words, the solubility of star polymers becomes better with increasing arm number if the total molecular weight is the same. We note that, if $\Theta / T_{\mathrm{c}}$ is plotted against $M_{\mathrm{w}}{ }^{-1 / 2}$, the literature data for eight-arm star PS in methylcyclohexane ${ }^{6,8}$ come much higher than the present data.

The spinodal is defined as the point at which $\left(\partial \mu_{0} / \partial \phi\right)$ or $K^{\prime} \phi / R_{0}$ vanishes (see eq 5 ). We determined it by extrapolating the $K^{\prime} / R_{0} v$ v. $T^{-1}$ plot for each solution to $K^{\prime} / R_{0}=0$. The filled circles in Figure 4 represent the spinodals thus obtained. The dashed line fitting these circles for each sample contacts the coexisting curve (solid line) at the critical point as is expected. All the spinodals from the present study are summarized in Table III.
Table III. Spinodal points for six-arm star polystyrene in cyclohexane

\begin{tabular}{cccccccc}
\hline \multicolumn{2}{c}{$6 \mathrm{~S} 17$} & \multicolumn{2}{c}{$6 \mathrm{S36}$} & \multicolumn{2}{c}{$6 \mathrm{~S} 85$} & \multicolumn{2}{c}{$6 \mathrm{~S} 190$} \\
\hline $\boldsymbol{\phi}$ & $T /{ }^{\circ} \mathrm{C}$ & $\phi$ & $T /{ }^{\circ} \mathrm{C}$ & $\phi$ & $T /{ }^{\circ} \mathrm{C}$ & $\phi$ & $T /{ }^{\circ} \mathrm{C}$ \\
\hline 0.1669 & 15.45 & 0.1310 & 20.11 & 0.0789 & 26.75 & 0.1000 & 24.82 \\
0.1361 & 16.29 & 0.1180 & 20.79 & 0.0607 & 26.70 & 0.0882 & 25.80 \\
0.1267 & 16.37 & 0.0796 & 21.31 & 0.0401 & 26.16 & 0.0804 & 26.43 \\
0.1008 & 16.54 & 0.0605 & 20.71 & 0.0204 & 23.67 & 0.0726 & 26.79 \\
0.1150 & 16.54 & 0.0428 & 19.59 & & & 0.0642 & 27.42 \\
0.0779 & 16.20 & 0.0354 & 18.82 & & & 0.0494 & 28.05 \\
0.0545 & 14.95 & 0.0219 & 16.03 & & & 0.0444 & 28.24 \\
0.0373 & 13.63 & & & & & 0.0321 & 28.51 \\
& & & & & & 0.0285 & 28.51 \\
& & & & & & 0.0217 & 28.15 \\
& & & & & & 0.0215 & 28.15 \\
& & & & & & 0.0168 & 27.15 \\
& & & & & & 0.0103 & 23.59 \\
\hline
\end{tabular}

\section{DISCUSSIONS}

Eq 5 allows the solvent chemical potential to be expressed in terms of the apparent second virial coefficient $J(\phi)(=J)$ as

$$
\frac{\mu_{0}-\mu_{0}^{\circ}}{R T}=-\frac{\phi}{P}-2 \int_{0}^{\phi} u J(u) d u
$$

where $\mu_{0}^{\circ}$ denotes $\mu_{0}$ in the infinite dilution limit. From this equation and the Gibbs-Duhem relation, the chemical potential of the solute $\mu_{1}$ is expressed as

$$
\frac{\mu_{1}-\mu_{1}{ }^{\infty}}{R T}=\ln \phi-\phi+2 P\left[\int_{0}^{\phi} J(u) d u-\int_{0}^{\phi} u J(u) d u\right]
$$

where

$$
\mu_{1}^{\infty}=\lim _{\phi \rightarrow 0}\left(\mu_{1}-R T \ln \phi\right)
$$

Thus, once we determine the functional form of $J(\phi)$, we can calculate $\mu_{0}$ and $\mu_{1}$.

Einaga et $a l .{ }^{11}$ expressed $J$ for linear PS in cyclohexane below $\Theta$ in an empirical manner as a sum of contributions from dilute and concentrated regions. Applying their equation to $J^{\prime}$ $\left(\equiv J-J_{0 \Theta}\right)$, we have

$$
J^{\prime}=\left(J_{0}{ }^{\prime}-J_{\mathrm{c} 0}\right) Q(\phi)+J_{\mathrm{conc}}(\phi)
$$

with

$$
Q(\phi)=\exp (-\phi / b)
$$

Here, $b$ is a function of $P$ and $T, J_{\text {conc }}(\phi)$ is a function of $\phi, T$, and $P$, and $J_{\mathrm{c} 0}$ is the $\phi$-independent term in $J_{\text {conc }}(\phi)$ (see eq 18).

Terao et $a l .{ }^{4}$ found that $J_{\text {conc }}(\phi)$ for both linear and four-arm star PS's in cyclohexane can be expressed by a common function of $\bar{\phi}\left(\equiv \phi / P^{0.1}\right)$ as

$$
J_{\text {conc }}(\phi)=J_{\mathrm{c} 0}+J_{\mathrm{c} 1} \bar{\phi}+J_{\mathrm{c} 2} \bar{\phi}^{2}
$$

with 


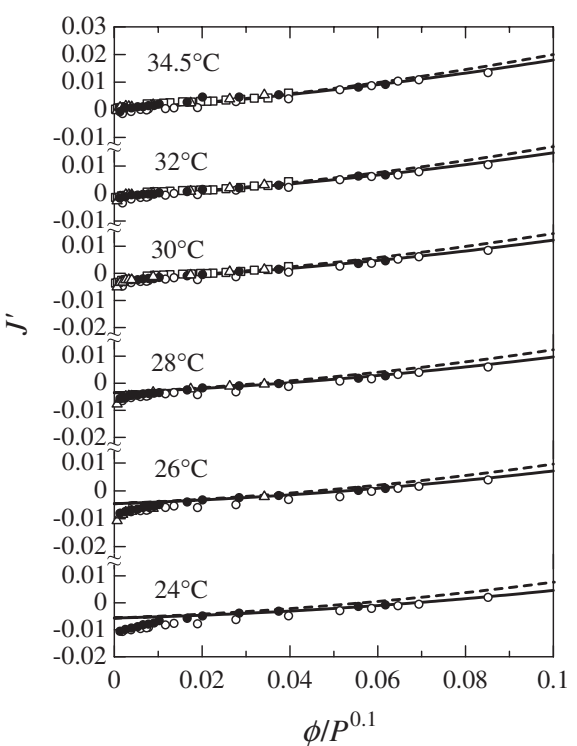

Figure 6. Plots of $J^{\prime}$ against $\phi / P^{0.1}$ at different temperatures. Symbols are as same as those used in Figure 3.

$$
\begin{gathered}
J_{\mathrm{c} 0}=0.17(1-\Theta / T)+0.3(1-\Theta / T)^{2} \\
J_{\mathrm{c} 1}=0.11+1.5(1-\Theta / T) \quad \text { (linear and four-arm star) } \\
J_{\mathrm{c} 2}=0.9+4.0(1-\Theta / T) \quad \text { (linear and four-arm star) }
\end{gathered}
$$

We note again that $J_{0 \Theta}=0$ for these polymers in the molecular weight range concerned.

In Figure 6, the present $J^{\prime}$ data plotted against $\phi / P^{0.1}$ are compared with the dashed lines representing $J_{\text {conc }}(\phi)$ (i.e., eq 18 with eqs $19-21)$, with the $\left(J_{0}{ }^{\prime}-J_{\mathrm{c} 0}\right) Q(\phi)$ term neglected. The agreement is not very bad, but as indicated by solid lines, closer agreement is obtained at high concentrations if eqs 20 and 21 are replaced, respectively, by

$$
\begin{aligned}
& J_{\mathrm{c} 1}=0.11+2.0(1-\Theta / T) \quad(\text { six-arm star }) \\
& J_{\mathrm{c} 2}=0.8+2.0(1-\Theta / T) \quad(\text { six-arm star })
\end{aligned}
$$

Thus, with these relations, the remaining unknown $b$ in $Q(\phi)$ was determined to be

$$
b=[2.0(1-\Theta / T)+1.0] / P^{1 / 2}
$$

so as to give the closest agreement between the calculated and observed $J\left(=J^{\prime}+J_{0 \Theta}\right)$ down to $\phi=0$. The solid lines in Figure 2 represent the calculated $J$ values. Their fits to the data points are satisfactory.

With the $J$ function obtained in this way, we computed the excess chemical potentials of the solvent and solute components through eqs 13 and 14 and then binodals, spinodals, and critical points. Figure 7 shows that the calculated and observed results for six-arm star PS are in fair agreement. In Figure 5, the solid curve closely fitting the unfilled circles for the six-arm star PS represents the calculated critical points and the dashed and dotdashed lines refer to linear and four-arm star PS's, respectively (the previous calculation ${ }^{4}$ ). The satisfactory description of the molecular weight dependence of $T_{\mathrm{c}}$ by the calculated lines ensures that our chemical potential functions are accurate.

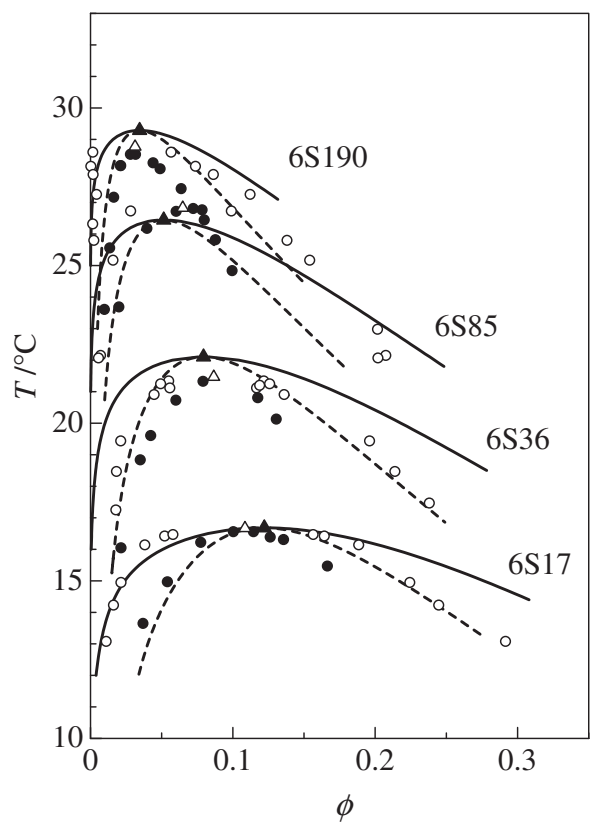

Figure 7. Comparison between observed and calculated phase diagrams. Solid lines, dashed lines, and filled triangles represent calculated binodal, spinodal, and critical points, respectively. Other symbols are as same as those used in Figure 4.

As mentioned in the Introduction, the difference between the phase diagrams of linear and four-arm star PS's stems from that in $J_{0}$, i.e., from the difference in the solvent chemical potential only in the dilute region. ${ }^{4}$ The present analysis shows that there exists no significant difference in $J_{0}$ between the six-arm and four-arm star PS's. Although the phase-separation behavior is rather similar between these two star polymers, the systematic difference in $T_{\mathrm{c}}^{-1}$ is apparent (Figure 5). The higher solubility of six-arm star PS as indicated by the larger $T_{\mathrm{c}}^{-1}$ in the figure is seemingly opposite to the smaller $J^{\prime}$ or $J_{\text {conc }}(\phi)$ values at high concentrations in Figure 6 (the solid line slightly below the dashed one). However, the higher $T_{\mathrm{c}}^{-1}$ (or higher solubility) of this star polymer results from the chain-end effect that leads $J(\phi)$ to exceed the value for the four-arm star polymer. In the present analysis, this significant end effect estimated from $A_{2 \Theta}$ is assumed to be independent of $\phi$, because no more detailed information is available.

\section{CONCLUSIONS}

The following conclusions may be derived from the present light scattering and phase separation studies on six-arm star PS in cyclohexane.

1. The reduced second virial coefficient $J_{0}$, corrected for the chain-end effect by subtraction of its contribution $J_{0 \Theta}$, is represented by a function of $(1-\Theta / T)$ almost regardless of molecular weight below $\Theta$ as is the case for linear and fourarm star PS's in cyclohexane.

2. When similarly corrected for this end effect, the contribution $J_{\text {conc }}(\phi)$ to the apparent second virial coefficient $J(\phi)$ at a given $T$ in a high concentration region is a function only of $\bar{\phi}$ 
$\left(=\phi / P^{0.1}\right)$, which is different slightly from that previously ${ }^{4}$ obtained for both linear and four-arm star PS's in cyclohexane. 3. The calculated phase diagrams agree fairly well with the observed ones, substantiating that the chemical potentials of the solvent and solute components derived from the $J(\phi)$ function are accurate.

4. The solubility of six-arm star PS higher than that of four-arm star PS in cyclohexane is attributable to the chain-end effect present over the dilute to concentrated region.

Acknowledgment. We thank Dr. Ken Terao of Osaka University for his help in the phase separation experiments.

Received: January 23, 2008

Accepted: March 29, 2008

Published: May 21, 2008

\section{REFERENCES}

1. J. M. G. Cowie, A. Horta, I. J. McEwen, and K. Prochazka, Polym. Bull., 1, 329 (1979).

2. S. Sato, M. Okada, and T. Nose, Polym. Bull., 13, 277 (1985).

3. H. Yokoyama, A. Takano, M. Okada, and T. Nose, Polymer, 32, 3218 (1991).

4. K. Terao, M. Okumoto, Y. Nakamura, T. Norisuye, and A. Teramoto, Macromolecules, 31, 6885 (1998).

5. M. L. Alessi, K. C. Bittner, and S. C. Greer, J. Polym. Sci., Part B: Polym. Phys., 42, 129 (2004).
6. D. T. Jacobs, C. I. Braganza, A. P. Brinck, A. B. Cohen, M. A. Lightfoot, C. J. Locke, S. J. Suddendorf, H. R. Timmers, A. L. Triplett, N. L. Venkataraman, and M. T. Wellons, J. Chem. Phys. 127, 124905 (2007).

7. P. J. Flory, "Principles of Polymer Chemistry," Cornell University Press, Ithaca, 1953.

8. G. Arya and A. Z. Panagiotopoulos, Macromolecules, 38, 10596 (2005).

9. J. Yang, C. Peng, H. Liu, Y. Hu, and J. Jiang, Fluid Phase Equilib. 244, 188 (2006).

10. Y. Einaga, S. Ohashi, Z. Tong, and H. Fujita, Macromolecules, 17, 527 (1984).

11. Y. Einaga, Z. Tong, and H. Fujita, Macromolecules, 18, 2258 (1985).

12. H. Fujita, "Polymer Solutions," Elsevier, Amsterdam, 1990.

13. M. Okumoto, Y. Tasaka, Y. Nakamura, and T. Norisuye, Macromolecules, 32, 7430 (1999).

14. M. Okumoto, Y. Iwamoto, Y. Nakamura, and T. Norisuye, Polym. J. 32, 422 (2000).

15. H. Yamakawa, Macromolecules, 25, 1912 (1992).

16. Y. Einaga, F. Abe, and H. Yamakawa, Macromolecules, 26, 6243 (1993).

17. T. Mizuno, K. Terao, Y. Nakamura, and T. Norisuye, Macromolecules, 38, 4432 (2005).

18. S. A. Antonenko and A. I. Sokolov, Phys. Rev. E, 51, 1894 (1995).

19. M. Nakata, N. Kuwahara, and M. Kaneko, J. Chem. Phys., 62, 4278 (1975).

20. M. Nakata, T. Dobashi, N. Kuwahara, M. Kaneko, and B. Chu, Phys. Rev. A, 18, 2683 (1978).

21. J. Hashizume, A. Teramoto, and H. Fujita, J. Polym. Sci., Polym. Phys. Ed., 19, 1405 (1981).

22. M. Tsuyumoto, Y. Einaga, and H. Fujita, Polym. J., 16, 229 (1984). 